\title{
Organ-specific autoantibodies and autoimmune diseases in juvenile systemic lupus erythematosus and juvenile dermatomyositis patients
}

\author{
Nadia E Aikawa*, Adriana A Jesus, Bernadete L Liphaus, Bernadete L Liphaus, Clovis A Silva, Magda C Sampaio, \\ Adriana M Sallum
}

From 2011 Pediatric Rheumatology Symposium sponsored by the American College of Rheumatology Miami, FL, USA. 2-5 June 2011

\section{Purpose}

The purpose of this study was to evaluate organ-specific autoantibodies and autoimmune diseases in JSLE and JDM patients.

\section{Methods}

Forty-one JSLE and 41 JDM patients were investigated for serum autoantibodies associated with autoimmune hepatitis, primary biliary cirrhosis, type 1 diabetes mellitus (T1DM), autoimmune thyroiditis, autoimmune gastritis and celiac disease. Patients with positive organ-specific antibodies were assessed for the presence of the respective organ-specific autoimmune diseases.

\section{Results}

Mean age at diagnosis was significantly higher in JSLE compared to JDM patients $(10.3 \pm 3.4$ vs. $7.3 \pm 3$.1years, $\mathrm{p}=0.0001$ ), whereas the mean disease duration was similar in both groups. The frequencies of organ-specific autoantibodies were similar between JSLE and JDM ( $>>0.05)$. Of note, a high prevalence of autoantibodies related to DM1 and autoimmune thyroiditis was observed in both groups ( $20 \%$ vs. $15 \%, \mathrm{p}=0.77$ and $24 \%$ vs. $15 \%, \mathrm{p}=0.41$; respectively). Higher frequencies of antinuclear antibody - ANA ( $93 \%$ vs. $59 \%, \mathrm{p}=0.0006)$, anti-dsDNA ( $61 \%$ vs. $2 \%$, $\mathrm{p}<0.0001)$, anti-Ro ( $35 \%$ vs. $0 \%, \mathrm{p}<0.0001)$, anti-Sm $(\mathrm{p}=0.01)$, anti-RNP $(\mathrm{p}=0.02)$, anti-La $(\mathrm{p}=0.03)$ and IgG $\mathrm{aCL}(\mathrm{p}=0.001)$ were observed in JSLE compared to JDM patients. Organ-specific autoimmune diseases were evidenced only in JSLE patients ( $24 \%$ vs. $0 \%, p=0.13$ ). Two JSLE patients had T1DM associated with Hashimoto thyroiditis and another had subclinical thyroiditis. Other JSLE patient had celiac disease diagnosis based on iron deficiency anaemia, presence of anti-endomysial antibody, duodenal biopsy compatible to celiac disease and response to a gluten-free diet.

\section{Conclusion}

Organ-specific diseases were observed solely in JSLE patients and required specific therapy. The presence of these antibodies recommends the evaluation of organspecific diseases and a rigorous follow-up of these patients.

\section{Disclosure}

Nadia E. Aikawa: None; Adriana A. Jesus: None; Bernadete L. Liphaus: None; Bernadete L. Liphaus: None; Clovis A. Silva: None; Magda C. Sampaio: None; Adriana M. Sallum: None.

Published: 13 July 2012

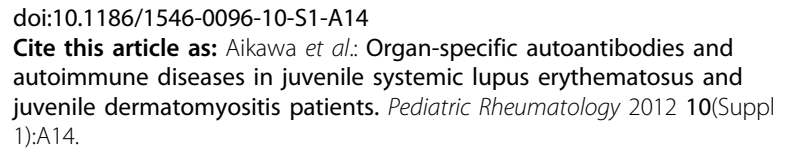

